\title{
BMJ Open Low-value clinical practices in injury care: a scoping review protocol
}

To cite: Moore L, Boukar KM, Tardif P-A, et al. Lowvalue clinical practices in injury care: a scoping review protocol. BMJ Open 2017;7:e016024. doi:10.1136/ bmjopen-2017-016024

- Prepublication history for this paper is available online. To view these files please visit the journal online (http://dx.doi. org/10.1136/bmjopen-2017016024).

Received 17 January 2017 Revised 28 March 2017 Accepted 21 April 2017

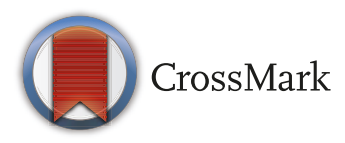

For numbered affiliations see end of article.

Correspondence to

Dr. Lynne Moore; lynne.moore@ fmed.ulaval.ca

\author{
Lynne Moore, ${ }^{1,2}$ Khadidja Malloum Boukar, ${ }^{1,2}$ Pier-Alexandre Tardif, ${ }^{2}$ \\ Henry T Stelfox, ${ }^{3}$ Howard Champion, ${ }^{4}$ Peter Cameron, ${ }^{5}$ Belinda Gabbe, ${ }^{6}$ \\ Natalie Yanchar, ${ }^{7}$ John kortbeek, ${ }^{8}$ François Lauzier, ${ }^{1,2}$ France Légaré, $^{9}$ \\ Patrick Archambault, ${ }^{9}$ Alexis F Turgeon ${ }^{9}$
}

\begin{abstract}
Introduction Preventable injuries lead to 200000 hospital stays, 60000 disabilities, and 13000 deaths per year in Canada with direct costs of $\$ 20$ billion. Overall, potentially unnecessary medical interventions are estimated to consume up to $30 \%$ of healthcare resources and may expose patients to avoidable harm. However, little is known about overuse for acute injury care. We aim to identify lowvalue clinical practices in injury care.

Methods and analysis We will perform a scoping review of peer-reviewed and non-peer-reviewed literature to identify research articles, reviews, recommendations and guidelines that identify at least one low-value clinical practice specific to injury populations. We will search Medline, EMBASE, COCHRANE central, and BIOSIS/Web of

\section{Strengths and limitations of this study}

Objective, rigorous and systematic identification of low-value clinical practices in injury care.

- Fill a major knowledge gap on medical overuse for acute injury care.

- Inform research priorities and the development of metrics to measure overuse.

- Represents a crucial step towards the deadoption of low-value clinical practices in acute injury care.

- For feasibility reasons, restricted to studies published since 2006.

- Scoping design means no appraisal of methodological quality-this will be evaluated in ensuing systematic reviews.
\end{abstract} Knowledge databases, websites of government agencies, professional societies and patient advocacy organisations, thesis holdings and conference proceedings. Pairs of independent reviewers will evaluate studies for eligibility and extract data from included articles using a prepiloted and standardised electronic data abstraction form.

Low-value clinical practices will be categorised using an extension of the Agency for Healthcare Research and Quality conceptual framework and data will be presented using narrative synthesis.

Ethics and dissemination Ethics approval is not required as original data will not be collected. This study will be disseminated in a peer-reviewed journal, international scientific meetings, and to knowledge users through clinical and healthcare quality associations. This review will contribute new knowledge on low-value clinical practices in acute injury care. Our results will support the development indicators to measure resource overuse and inform policy makers on potential targets for deadoption in injury care.

\section{INTRODUCTION}

Preventable injuries represent a leading cause of death, disability and healthcare expenditures. ${ }^{1}$ Canadian injury deaths increased from 13000 in 2004 to 16000 in 2010 while costs increased by $35 \%$ and are projected to reach $\$ 75$ billion by 2035 . ${ }^{1}$ The huge burden of injury and evidence of variation in injury outcomes across healthcare providers ${ }^{2-4}$ demonstrate that efforts to optimise processes of care have the potential to yield major dividends.
Emphasis on adherence to recommended processes of care and rapid innovation in imaging and therapeutic techniques has led to an exponential rise in the use of tests and treatments that are not supported by evidence and/or could expose patients to unnecessary harm, ${ }^{5}$ referred to here as low-value clinical practices. ${ }^{6-13}$ Examples include whole body CT for minor or single-system injury and steroid administration following severe traumatic brain injury. ${ }^{5}$ Overall, unnecessary clinical processes have been estimated to consume up to $30 \%$ of healthcare resources $^{8101214}$ but little is known about healthcare overuse in the context of injury care. Importantly, unnecessary diagnostic or therapeutic interventions may expose patients to harm through adverse events (eg, inappropriate surgery that could result in surgical infection) and delays to effective therapy (eg, extensive imagery in a patient with traumatic brain injury prior to transfer to a level I trauma centre)..$^{6-8} 1012$ Interventions targeting the deadoption of low-value clinical practices have the potential to reduce waste and improve patient outcomes. ${ }^{1315}$

Audit and feedback using quality indicators has been shown to impact favourably on 
healthcare outcomes by improving clinical practices. ${ }^{16}$ However, $94 \%$ of indicators address lack of adherence to recommended processes of care (underuse). ${ }^{17} 18$ Indicators designed to monitor the use of potentially unnecessary tests and interventions (overuse) are needed to ensure physicians ask themselves not only 'am I doing enough?' but also 'am I doing too much?' While interventions on a patient level may well be justified by unmeasured risk factors or patient/family preferences, information on systematic provider variations in the use of low-value clinical practices after risk stratification can be used to inform quality improvement initiatives. ${ }^{3}$ Physicians report overusing resources for fear of legal actions but also because of lack of guidelines on low-value clinical practices. ${ }^{10-12}{ }^{19}$ Choosing Wisely has developed a list of commonly used tests or procedures whose necessity should be questioned. ${ }^{5}$ However, few apply to injury care and most are based uniquely on expert consensus. Previous reviews aiming to identify low-value clinical practices have not been specific to injury but have underlined the importance of targeting diagnostic groups to improve feasibility and actionability of results. ${ }^{132-23}$ We urgently need to identify low-value clinical practices in injury care as a first step towards evaluating the problem of overuse in this important patient population.

We aim to identify low-value clinical practices in injury care that can be used to inform the development of quality indicators to measure resource overuse.

\section{METHODS AND ANALYSIS}

The protocol is structured in six stages following published guidelines for scoping reviews. ${ }^{24}$ As this is a scoping review intended to generate rather than verify hypotheses, methods may be modified as the review progresses. ${ }^{25-28}$

\section{Identify research questions}

Using an iterative approach, the interdisciplinary and intersectorial project steering committee comprising clinicians (trauma surgeons, emergency physicians, critical care physicians, prehospital personnel), allied health professionals (nurses, physical therapists) and policy makers and decision makers (trauma programme leaders, representatives of trauma accreditation agencies) identified the following research question for our scoping review: Which diagnostic or therapeutic interventions are considered low-value in acute injury care?

\section{Identify relevant studies}

\section{Eligibility criteria}

We will include research articles, reviews, recommendations and guidelines that identify at least one low-value clinical practice specific to injury populations. As stated above, low-value clinical practices are defined as commonly used tests and treatments (eg, laboratory tests, imaging, transfusions, surgeries) that are not supported by evidence and/or could expose patients to unnecessary harm. ${ }^{5}$ We will include studies on clinical practices specific to intrahospital acute care (in the emergency department or following hospital admission). We will include all study designs, for example, studies that evaluate the effectiveness of an intervention, measure the prevalence of low-value practices, propose a guideline/recommendation on low-value clinical practices, or evaluate the efficacy of an intervention for the deadoption of a low-value clinical practice. We will include research and non-research documents based on emergency department (ED) admissions and inpatient hospitalisations for injury. Studies on general injury admissions or on admissions for specific injury types (eg, traumatic brain injury, thoracoabdominal injury, orthopaedic injuries) or age groups (paediatric, adult, geriatric) will be included. The clinical practice could be low-value for all patients or for specific patient subgroups. We will exclude the following: (1) studies on pharmaceutical agents (blood products and their derivatives will be included), (2) studies based exclusively on populations with combat injuries, isolated fractures following low falls, burns, bites, foreign bodies or late effects of injuries, (3) case reports, (4) studies on clinical practices in injury prevention and the postacute phases of injury care (eg, rehabilitation, community maintenance). To ensure the feasibility of the review, will limit the search to documents published in English since January, 2006. The study will cover publications appearing between 1 January 2006 up to a maximum of 6 months before submission of the final manuscript.

\section{Information sources}

We will systematically search the following:

1) Medical Literature Analysis and Retrieval System Online (MEDLINE), Excerpta Medica dataBASE (EMBASE), Cochrane Central, Biosciences Information Service (BIOSIS)/Web of Knowledge, ClinicalTrials and International Standard Randomised Controlled Trials Number (ISRCTN) databases from their inception up to a maximum of 9 months before publication submission.

2) Thesis repositories including Thesis portal Canada, Electronic Thesis Online Service (EThOS), Digital Access to Research Theses (DART)-Europe E-Theses Portal, the National Library of Australia's Trove and ProQuest Dissertations \& Theses Global.

3) Websites of:

a. Healthcare quality organisations including the WHO, National Institute for Health and Care Excellence, National Association for Healthcare Quality, National Quality Forum, Lown Institute, Agency for Healthcare Research and Quality, Choosing Wisely, Canadian Institutes for Health Information, Australasian Association for Quality in Healthcare.

b. Injury organisations including the American College of Surgeons, Trauma Association of Canada, International Association for Trauma Surgery and 
Intensive Care, Australasian Trauma Society, Trauma Audit Research Network, American Association for the Surgery of Trauma, Eastern Association for the Surgery of Trauma, American Trauma Society, British Trauma Society, Orthopaedic Trauma Association, Western Trauma Association, Trauma.org, The Society of Trauma Nurses, International Trauma Anaesthesia and Critical Care Society, BrainTrauma Foundation.

c. Patient advocacy organisations including Safer Healthcare Now!

References of included articles will then be screened for any further eligible studies.

\section{Search strategy}

Using Cochrane guidelines ${ }^{29}$ we will develop a rigorous systematic search strategy in collaboration with an information specialist. We will use combinations of search terms under the themes injury and low-value clinical practices (see table 1 for a preliminary search strategy) using keywords elaborated by the project steering committee comprising clinical and methodological experts. Our search strategy will be developed for Medline (Medical Subject Headings; MeSH) and EMBASE (Embase tree; EMTREE) and will then be adapted to the other databases. The information specialist will peer review the search strategy using the Peer Review of Electronic Search Strategies checklist. ${ }^{30}$

\section{Select studies}

\section{Data management}

We will organise citations using EndNote (V.X7.0.1, New York City: Thomson Reuters, 2011). We will identify and remove duplicates by electronic and manual screening. In the case of multiple publications based on the same data, we will include the study based on the largest sample size in analyses.

\section{Selection process}

Pairs of reviewers (two of three reviewers LM, KMB, P-AT) will first screen titles and abstracts and will then evaluate full-text publications to assess final eligibility. We will evaluate agreement between the three reviewers on eligibility using the first 500 citations (or more if deemed necessary). If necessary, we will then clarify inclusion criteria and repeat the process until acceptable inter-rater agreement is attained. We will settle any further disagreement on study eligibility by consensus and a fourth reviewer will adjudicate if necessary (AFT). If information on eligibility is unavailable or unclear, study authors will be contacted to clarify.

\section{Chart material}

\section{Data collection}

A standard electronic data abstraction form and a detailed instruction manual will be developed and piloted on a representative sample of five publications (table 2). Pairs of reviewers (LM, KMB, P-AT) with methodological and content expertise will independently extract information on the study design (systematic review with meta-analysis, randomized controlled trial (RCT), evidence-based guidelines), setting (country, year, language, funding), population (eg, age, injury type and severity), low-value clinical practices, and primary outcomes when appropriate (eg, mortality, morbidity, resource use, costs). Any discrepancies between reviewers will be resolved by consensus and a fourth reviewer will adjudicate if necessary (AFT). We will contact study authors if important information is missing or requires clarification using up to three email attempts over 1 month to all listed authors.

\section{Collate, summarise and report on results}

Criteria will be classified according to the type of low-value practice and type of service based on the conceptual framework proposed by Chan et al (table 3). ${ }^{20}$ This framework is an extension of conceptualisations proposed by the Agency for Healthcare Research and Quality ${ }^{31}$ and Fisher and Wennberg. ${ }^{32}$ Classifications will be conducted independently by two reviewers (KMB, P-AT) and then checked independently by a third reviewer (LM). Any disagreements will be adjudicated by a third reviewer (AFT). As is common in scoping reviews, methodological quality of included studies will not be evaluated. ${ }^{25}$

\section{Consultation}

We will consult our project advisory committee comprising healthcare practitioners (emergency physician, intensivist, trauma surgeon, neurosurgeon, orthopaedic surgeon), allied health professionals (nurses, physical therapists), policy makers and decision makers (representatives of the Québec National Institute of Health Care Excellence, Québec Ministry of Health and Choosing Wisely Canada), and patient/family representatives. The objectives of the consultation will be threefold: (1) identify any further references (2) obtain feedback on the interpretation and presentation of results, (3) identify opportunities for knowledge transfer.

\section{CONCLUSIONS}

This scoping review will fill an important gap on low-value clinical practices in the clinical area of acute injury care. This review is a component of the Canadian Programme for Monitoring Overuse in Injury Care. Our overarching goal is to develop indicators that can be used to measure resource overuse in injury care and inform the deadoption of low-value clinical practices.

Review results will be used to inform the Canadian Program for Monitoring Overuse in Injury Care, a 5-year research programme which has received peer-reviewed federal funding (Canadian Institutes for Health Research Foundation grant). The second stage aims to build the evidence base for low-value clinical practices identified in this review using a series of systematic reviews. We will then conduct a RAND/ UCLA (Research and Development / University of California at Los Angeles) expert consensus study based on the best available evidence to develop indicators, 


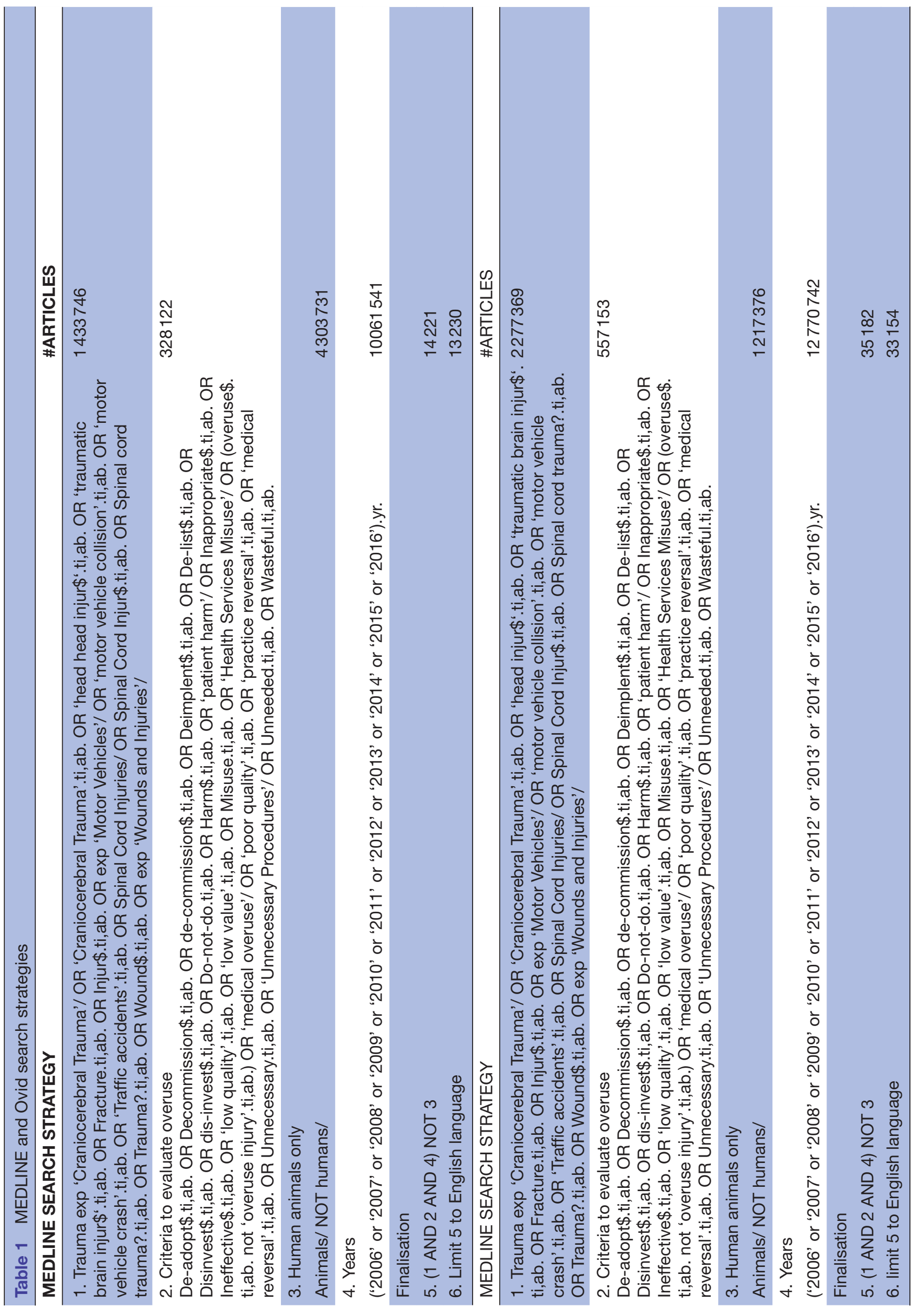

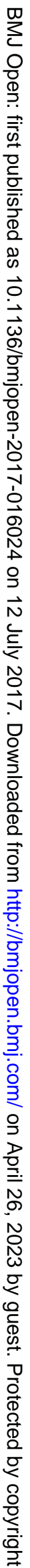


Table 2 Data collection form*

Title of review

Low-value clinical practices in injury care: a scoping review

Reviewer

Date of review

Form version

$1.0(2017-01-16)$

Contact with author

Date, reason, resolved

Notes

Study identification

Report number

Last name first author-year of the reference

Study number

Last name first author-year of the primary reference

Title

Author(s)

Source 1

$\square$ research article $\square$ abstract $\square$ conference proceeding

$\square$ non-research article $\square$ review $\square$ guideline $\square$ recommendation other (eg, unpublished data):

Source 2

$\square$ MEDLINE $\square$ EMBASE $\square$ Cochrane $\square$ BIOSIS $\square$ ClinicalTrials $\square$ ISRCTN $\square$ Thesis repository $\square$

Website $\square$ Reference listing

Other (grey literature):

Year

Volume

Page (start-end)

Contact

Country

Language

Funding

$\square$ NA

Notes

Eligibility of study in the review
Inclusion criteria
$\square$ Injury population
$\square$ potentially low-value clinical practice

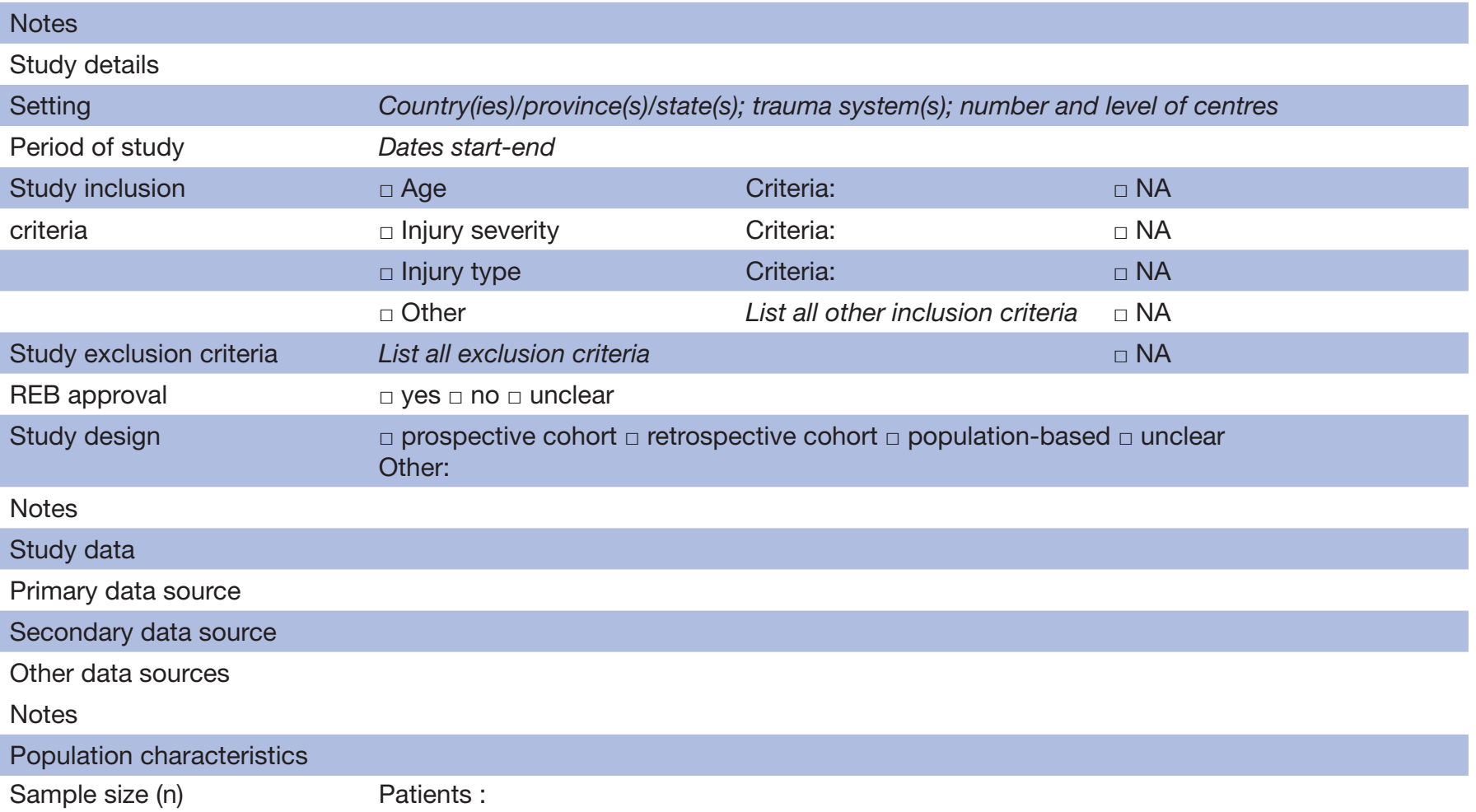




\begin{tabular}{|c|c|}
\hline Age & e.g. $n(\%) \geq 65$ yoa, mean (SD), median (quartiles) \\
\hline Gender & $n(\%)$ male \\
\hline Injury mechanism & e.g. $n(\%)$ penetrating, motor vehicle collision \\
\hline Injury severity & e.g. $n(\%)$ Injury Severity Score>15, Maximum Abbreviated Injury Scale score>3 \\
\hline Injury type & e.g. $n(\%)$ traumatic brain; spinal cord; thoracoabdominal; orthopaedic; multisystem blunt \\
\hline \multicolumn{2}{|l|}{ Notes } \\
\hline \multicolumn{2}{|c|}{ Low-value clinical practice (one per practice) } \\
\hline \multicolumn{2}{|l|}{ Practice } \\
\hline Type of practice & $\begin{array}{l}\square \text { consultation } \square \text { screening } \square \text { diagnostic procedure } \square \text { monitoring } \square \text { therapeutic procedure } \\
\text { Other: }\end{array}$ \\
\hline Type of overuse & $\begin{array}{l}\square \text { inappropriate for a specified clinical indication } \\
\square \text { inappropriate for clinical indication in a specific population } \\
\square \text { excessive service intensity or sophistication given expected clinical benefit } \\
\square \text { inappropriate for clinical indication in a specific population } \\
\square \text { excessive frequency of service given expected clinical benefit } \\
\text { Other: }\end{array}$ \\
\hline Frequency & e.g. $n(\%)$ \\
\hline
\end{tabular}

NA: not available, MEDLINE: Medical Literature Analysis and Retrieval System Online, EMBASE: Excerpta Medica database, BIOSIS:

Biosciences Information Service, ISRCTN: International Standard Randomised Controlled Trials Number

*Adapted from Cochrane Consumer and Communication Review Group Data extraction template.

a multicentre retrospective cohort study to derive and validate metrics for the indicators and a cluster randomised controlled study to evaluate the effectiveness of indicators in an audit-feedback intervention. This review therefore represents a first step towards developing valid and reliable metrics to measure potentially unnecessary or harmful processes specific to acute care following injury. These metrics will enable us to advance knowledge on the prevalence of overuse, its determinants and its impact on patient outcomes.
This knowledge will provide a solid basis for the development of interventions targeting deadoption such as shared decision making tools. Such interventions have the potential to reduce costs, delays and unnecessary hospital days and increase resource availability. They may also improve patient outcomes through a reduction in exposure to adverse events and delays to care.

Table 3 Framework for classifying low-value clinical practices

\begin{tabular}{ll}
\hline & \multicolumn{1}{c}{ Type of process } \\
\hline Type of overuse & $\begin{array}{l}\text { Admission, } \\
\text { transfer }\end{array} \quad$ Consultation Screening Diagnostic $\quad$ Monitoring Therapeutic \\
\hline $\begin{array}{l}\text { Inappropriate for a specified clinical } \\
\text { indication* }\end{array}$ & \\
Inappropriate for clinical indication in \\
a specific population† \\
$\begin{array}{l}\text { Excessive service intensity or sophistication } \\
\text { given expected clinical benefitł }\end{array}$
\end{tabular}

Excessive frequency of service given

expected clinical benefit§

\footnotetext{
${ }^{*}$ Specific clinical situations or indications for which a service is considered inappropriate or of questionable clinical value (eg, antibiotics for acute bronchitis).

†Services that may be appropriate for a specific population, such as a high-risk population, but is inappropriate or of negligible clinical benefit when applied to other, particularly lower-risk populations (eg, cardiac stress imaging for initial detection and risk assessment in asymptomatic, low coronary heart disease risk individuals).

$\ddagger$ More expensive or intensive services with marginal clinical benefits when less expensive or less intensive, but equally effective alternatives, are available (eg, combined, with and without contrast, abdominal CT scans when only one scan is necessary).

§Repeating tests too frequently when the probability of observing clinically important change is low and can increase costs and patient exposure to risks unnecessarily (eg, frequency of follow-up or monitoring).

Source, Chan et al. ${ }^{20}$
} 
Author affiliations

${ }^{1}$ Department of Social and Preventative Medicine, Université Laval, Québec (Qc), Canada

${ }^{2}$ Population Health and Optimal Health Practices Research Unit, Trauma Emergency - Critical Care Medicine, Centre de Recherche du CHU de Québec (Hôpital de l'Enfant-Jésus), Université Laval, Québec, Canada ${ }^{3}$ Departments of Critical Care Medicine, Medicine and Community Health Sciences, 0'Brien Institute for Public Health,University of Calgary, Calgary, Canada

${ }^{4}$ Departement of Surgery, U Health Sciences, Maryland, USA

${ }^{5}$ The Alfred Hospital,Monash University, Melbourne, Australia

${ }^{6}$ School of Public Health and Preventive Medicine,Monash University, Melbourne, Australia

${ }^{7}$ Department of Surgery, Dalhousie University, Halifax, Canada

${ }^{8}$ Department of Surgery, University of Calgary, Calgary, Canada

${ }^{9}$ Department of Medicine, Université de Montréal, Québec, Canada

Contributors LM led the development of the protocol and drafted the manuscript. She acts as guarantor for the review. KMB contributed to the development of research objectives and inclusion criteria, contributed to the elaboration of keywords, the search strategy and the data extraction form, critically revised and approved the final version of the manuscript. P-AT contributed to the elaboration of keywords, developed and tested the search strategy, drafted parts of the methods, critically revised and approved the final version of the manuscript. HTS contributed to the development of research objectives, inclusion criteria, the search strategy and the extraction form, developed keywords, revised the manuscript and approved the final version. Flée contributed to the development of research objectives, study definitions, inclusion criteria, and the extraction form, developed keywords, revised the manuscript and approved the final version. $\mathrm{HC}$ contributed to the development of research objectives and inclusion criteria, elaborated keywords, validated the data extraction form, critically revised the manuscript and approved the final version. PC elaborated inclusion and exclusion criteria and keywords, contribution to the development of the conceptual framework and concept definitions, revised the manuscript and approved the final version. BG elaborated inclusion and exclusion criteria and keywords, revised the manuscript and approved the final version. NY contributed to working definitions, developed keywords, revised the manuscript and approved the final version. JK contributed to working definitions, revised the manuscript and approved the final version. Fç contributed to developing keywords, validated the search strategy and the data extraction form, revised the manuscript and approved the final version. MC validated the search strategy and the data extraction form, revised the manuscript and approved the final version. PA contributed to working definitions, developed keywords, revised the manuscript and approved the final version. AFT elaborated inclusion criteria and clinically significant outcomes, validated the search strategy, elaborated keywords, revised the manuscript and approved the final version extraction form.

Funding This research is funded by the Canadian Institutes of Health Research (Foundation grant, \#353374) andthe Fonds de Recherche du Québec - Santé (career award, LM).

\section{Competing interests None declared.}

Patient consent Detail has been removed from these case descriptions to ensure anonymity. The editors and reviewers have seen the detailed information available and are satisfied that the information backs up the case the authors are making.

Provenance and peer review Not commissioned; externally peer reviewed.

Open Access This is an Open Access article distributed in accordance with the Creative Commons Attribution Non Commercial (CC BY-NC 4.0) license, which permits others to distribute, remix, adapt, build upon this work non-commercially, and license their derivative works on different terms, provided the original work is properly cited and the use is non-commercial. See: http://creativecommons.org/ licenses/by-nc/4.0/

(C) Article author(s) (or their employer(s) unless otherwise stated in the text of the article) 2017. All rights reserved. No commercial use is permitted unless otherwise expressly granted.

\section{REFERENCES}

1. The cost of injury in Canada. Parachute Canada. 2015 http://www. smartrisk.ca/index.php/burden (accessed 17 Jan 2017).
2. Birkmeyer JD, Reames BN, McCulloch P, et al. Understanding of regional variation in the use of surgery. Lancet 2013;382:1121-9.

3. Fisher ES, Wennberg DE, Stukel TA, et al. The implications of regional variations in Medicare spending. part 2: health outcomes and satisfaction with care. Ann Intern Med 2003;138:288-98.

4. Moore L, Evans D, Hameed SM, et al. Mortality in Canadian Trauma Systems: a Multicenter Cohort Study. Ann Surg 2017;265:212-7.

5. Choosing wisely Canada. 2015 http://www.choosingwiselycanada. org/ (accessed 16 jan 2017).

6. Berwick DM, Hackbarth AD. Eliminating waste in US health care. JAMA 2012;307:1513-6.

7. Boat TF, Chao SM, O'Neill PH. From waste to value in health care. JAMA 2008;299:568-71.

8. Reilly BM, Evans AT. Much ado about (doing) nothing. Ann Intern Med 2009;150:270-1.

9. Morgan DJ, Dhruva SS, Wright SM, et al. Update on Medical Overuse: a Systematic Review. JAMA Intern Med 2016;176:1687-92.

10. Overuse 101.The Lown Institute. 2017. http://lowninstitute.org/learn/ overuse-101/

11. Berwick DM. Avoiding overuse-the next quality frontier. Lancet 2017.

12. Brownlee S, Chalkidou K, Doust J, et al. Evidence for overuse of medical services around the world. Lancet 2017.

13. Saini V, Brownlee S, Elshaug AG, et al. Addressing overuse and underuse around the world. Lancet 2017.

14. Physicians taking lead on appropriateness of care. Secondary Physicians taking lead on appropriateness of care. https://www. cma.ca/En/Pages/Physicians-taking-lead-appropriateness-care. aspx

15. Niven DJ, Mrklas KJ, Holodinsky JK, et al. Towards understanding the de-adoption of low-value clinical practices: a scoping review. BMC Med 2015;13:255.

16. Ivers N, Jamtvedt G, Flottorp S, et al. Audit and feedback: effects on professional practice and healthcare outcomes. Cochrane Database Syst Rev 2012;6:CD000259.

17. Newton EH, Zazzera EA, Van Moorsel G, et al. Undermeasuring overuse--an examination of National Clinical Performance measures. JAMA Intern Med 2015;175:1709-11.

18. Mathias JS, Baker DW. Developing quality measures to address overuse. JAMA 2013;309:1897-8.

19. Emanuel EJ, Fuchs VR. The perfect storm of overutilization. JAMA 2008;299:2789-91.

20. Chan KS, Chang E, Nassery N, et al. The state of overuse measurement: a critical review. Med Care Res Rev 2013;70:473-96.

21. de Vries EF, Struijs JN, Heijink R, et al. Are low-value care measures up to the task? A systematic review of the literature. BMC Health Serv Res 2016;16:405.

22. Elshaug AG, Rosenthal MB, Lavis JN, et al. Levers for addressing medical underuse and overuse: achieving high-value health care. Lancet 2017.

23. Segal JB, Bridges JF, Chang HY, et al. Identifying possible indicators of systematic overuse of health care procedures with claims data. Med Care 2014;52:157-63.

24. Arksey H, O'Malley L. Scoping studies: towards a methodological framework. Int J Soc Res Methodol 2005;8:19-32.

25. University of Adelaide. Methodology for JBI scoping reviews. The Joanna Briggs Institute. 2015 http://joannabriggs.org/assets/docs/ sumari/Reviewers-Manual_Methodology-for-JBI-Scoping-Reviews 2015 v2.pdf (accessed 27 Jan 2017).

26. Levac D, Colquhoun H, O'Brien KK. Scoping studies: advancing the methodology. Implement Sci 2010;5:69.

27. Peters MD, Godfrey $\mathrm{CM}$, Khalil $\mathrm{H}$, et al. Guidance for conducting systematic scoping reviews. Int J Evid Based Healthc 2015;13:141-6.

28. Tricco AC, Lillie E, Zarin W, et al. A scoping review on the conduct and reporting of scoping reviews. BMC Med Res Methodol 2016;16:15

29. Lefebvre C, Manheimer E, Glanville J, et al. Searching for studies. In: Higgins JPT, Green S, eds. Cochrane handbook for systematic reviews of interventions. 5.1.0 ed. Cochrane Collaboration, 2011.

30. Sampson M, McGowan J, Cogo E, et al. An evidence-based practice guideline for the peer review of electronic search strategies. $J$ Clin Epidemiol 2009;62:944-52.

31. Agency for Healthcare Research and Quality. Health care efficiency measures: identification, categorization, and evaluation. $2008 \mathrm{https}: / /$ archive.ahrq.gov/research/findings/final-reports/efficiency/efficiency. pdf (accessed 15 Dec 2016).

32. Wenneberg International Collaborative. http://www. wennbergcollaborative.org/ (accessed 4 Jan 2017). 